\title{
The mitochondrial calcium uniporter underlies metabolic fuel preference in skeletal muscle
}

\author{
Jennifer Q. Kwong, ${ }^{1,2}$ Jiuzhou Huo, ${ }^{1}$ Michael J. Bround, ${ }^{1}$ Justin G. Boyer, ${ }^{1}$ Jennifer A. Schwanekamp, ${ }^{1}$ \\ Nasab Ghazal, ${ }^{2}$ Joshua T. Maxwell, ${ }^{2}$ Young C. Jang, ${ }^{3}$ Zaza Khuchua, ${ }^{1,4}$ Kevin Shi, ${ }^{5}$ Donald M. Bers, ${ }^{6}$ \\ Jennifer Davis, ${ }^{5}$ and Jeffery D. Molkentin ${ }^{1,7}$ \\ 'Department of Pediatrics, Cincinnati Children's Hospital Medical Center, University of Cincinnati, Cincinnati, Ohio, USA. \\ ${ }^{2}$ Department of Pediatrics, Division of Cardiovascular Biology, Emory University School of Medicine, Atlanta, Georgia, \\ USA. ${ }^{3}$ School of Biological Sciences, Georgia Institute of Technology, Atlanta, Georgia, USA. ${ }^{4}$ Sechenov University, \\ Moscow, Russia. ${ }^{5}$ Department of Bioengineering, University of Washington, Seattle, Washington, USA. ${ }^{6}$ Department of \\ Pharmacology, University of California, Davis, California, USA. 'Howard Hughes Medical Institute, Cincinnati Children's \\ Hospital Medical Center, Cincinnati, Ohio, USA.
}

The mitochondrial $\mathrm{Ca}^{2+}$ uniporter (MCU) complex mediates acute mitochondrial $\mathrm{Ca}^{2+}$ influx. In skeletal muscle, $\mathrm{MCU}$ links $\mathrm{Ca}^{2+}$ signaling to energy production by directly enhancing the activity of key metabolic enzymes in the mitochondria. Here, we examined the role of MCU in skeletal muscle development and metabolic function by generating mouse models for the targeted deletion of $\mathrm{Mcu}$ in embryonic, postnatal, and adult skeletal muscle. Loss of Mcu did not affect muscle growth and maturation or otherwise cause pathology. Skeletal muscle-specific deletion of Mcu in mice also did not affect myofiber intracellular $\mathrm{Ca}^{2+}$ handling, but it did inhibit acute mitochondrial $\mathrm{Ca}^{2+}$ influx and mitochondrial respiration stimulated by $\mathrm{Ca}^{2+}$, resulting in reduced acute exercise performance in mice. However, loss of Mcu also resulted in enhanced muscle performance under conditions of fatigue, with a preferential shift toward fatty acid metabolism, resulting in reduced body fat with aging. Together, these results demonstrate that MCU-mediated mitochondrial $\mathrm{Ca}^{2+}$ regulation underlies skeletal muscle fuel selection at baseline and under enhanced physiological demands, which affects total homeostatic metabolism.

Authorship note: JQK and JH contributed equally to this work.

Conflict of interest: The authors have declared that no conflict of interest exists.

License: Copyright 2018, American Society for Clinical Investigation.

Submitted: April 17, 2018

Accepted: October 4, 2018

Published: November 15, 2018

Reference information: JCI Insight. 2018;3(22):e121689. https://doi.org/10.1172/jci. insight.121689.

\section{Introduction}

In skeletal muscle $\mathrm{Ca}^{2+}$ directly mediates excitation-contraction coupling as well as stimulating mitochondrial energy production. Within the myofiber, mitochondria and the sarcoplasmic reticulum (SR) are tethered together whereby SR $\mathrm{Ca}^{2+}$ release directly augments mitochondrial $\mathrm{Ca}^{2+}$ influx for contraction-metabolic coupling (1). Mitochondrial $\mathrm{Ca}^{2+}$ influx serves as a rapid signal for the allosteric activation of select matrix dehydrogenases of the tricarboxylic acid (TCA) cycle, as well as the mitochondrial ATP synthase, to collectively enhance energy output $(2,3)$.

The mitochondrial $\mathrm{Ca}^{2+}$ uniporter (MCU) complex comprises a central $\mathrm{Ca}^{2+}$-transducing pore made of MCU multimers $(4,5)$ and regulatory elements, including MICU1, MICU2, EMRE, SLC25a23, and MCUb (6-11), which facilitates the voltage-dependent import of $\mathrm{Ca}^{2+}$ into the mitochondrial matrix (12). Recent studies using mouse models of both global deletion and targeted cardiac-specific deletion of Mcu show its importance in mediating mitochondrial $\mathrm{Ca}^{2+}$ import and mitochondrial energy production (13-15). However, the MCU complex appears to preferentially regulate acute mitochondrial $\mathrm{Ca}^{2+}$ influx, such as with the fight-or-flight response. For example, cardiac-specific deletion of $M c u$ in mice showed that mitochondria still loaded with $\mathrm{Ca}^{2+}$ in the long term and that only acute modes of $\mathrm{Ca}^{2+}$ influx and metabolic coupling were compromised (14). Indeed, with prolonged stimulation, $\mathrm{Mcu}$-deficient cardiomyocytes and hearts no longer showed a defect in mitochondrial respiration augmentation (14).

There is evidence that the MCU plays a role in skeletal muscle growth and homeostasis. For example, global Mcu gene-deleted mice are growth retarded and show constitutive inhibition of the pyruvate dehydrogenase complex in skeletal muscle (13). MCU was also suggested to directly regulate cellular growth 
pathways because viral-mediated overexpression of MCU in young mice caused muscle hypertrophy, whereas viral-mediated shRNA knock down caused atrophy (16). These studies link MCU to metabolism and growth in skeletal muscle.

Here, we investigated the role of MCU in skeletal muscle development and function using mice with skeletal muscle-specific deletion of the Mcu gene initiated at embryonic, postnatal, and adult stages. We show that while deletion of $\mathrm{Mcu}$ did not alter skeletal muscle growth, it did alter metabolic substrate preference with enhanced fatty acid usage under stress, which affected muscle performance and exercise dynamics.

\section{Results}

Muscle-specific deletion of Mcu impairs acute mitochondrial $\mathrm{Ca}^{2+}$ influx. Constitutive impairment of MCU function in $\mathrm{Mcu}$ global gene-deleted mice caused a significant reduction in body weight and muscle fiber size (13). To explore the specific role of MCU-mediated mitochondrial $\mathrm{Ca}^{2+}$ signaling in skeletal muscle function, we developed a skeletal muscle-specific loss-of-function model in which $M c u$-loxP-targeted mice $\left(M c u^{f l f f}\right)$ were crossed to gene-targeted mice expressing a Cre recombinase under the control of the Myod locus (MyoD-Cre; Figure 1A) (17). Mcut/f-MyoD-Cre animals were viable and born the expected Mendelian frequencies (data not shown). Mitochondrial lysates isolated from quadriceps muscles were analyzed by Western blot analysis, which showed a near complete loss of MCU protein in $M c t^{f / f-M y o D-C r e}$ animals compared with $M c u^{f l f l}$ controls (Figure 1B).

Measurements of total $\mathrm{Ca}^{2+}$ levels in muscle mitochondria isolated from $M c u^{f l / f-M y o D-C r e}$ and $M c u^{f l / f l}$ animals using an o-cresolphthalein assay showed no change in basal mitochondrial $\mathrm{Ca}^{2+}$ content (Figure $1 \mathrm{C}$ ). Indeed, Rhod-2 AM loading of living permeabilized flexor digitorum brevis (FDB) myofibers, under conditions that only produce mitochondrial fluorescence that is $\mathrm{Ca}^{2+}$ sensitive, also showed no baseline differences in resting mitochondrial $\mathrm{Ca}^{2+}$ levels among $\mathrm{Mcu}^{f / f l}$, MyoD-Cre, and $M c u^{f / f-M y o D-C r e}$ genotypes (see Supplemental Figure 1A; supplemental material available online with this article; https://doi.org/10.1172/ jci.insight.121689DS1). Acute mitochondrial $\mathrm{Ca}^{2+}$ uptake in response to a $\mathrm{Ca}^{2+}$ bolus was measured in Rhod-2 AM-loaded FDB myofibers that were saponin permeabilized, which showed increased Rhod-2 fluorescence in $M c u^{f l f l}$ control myofibers but to a significantly lesser degree in Mcu ${ }^{f / f-M y o D-C r e}$ myofibers lacking MCU (Figure 1D and Supplemental Figure 1B). Additionally, mitochondrial $\mathrm{Ca}^{2+}$ uptake was assessed in purified skeletal muscle mitochondria isolated from 2-month-old animals using the $\mathrm{Ca}^{2+}$ sensor Calcium Green-5N. While $M c u^{f / f l}$ and MyoD-Cre control mitochondria displayed decreases in calcium green fluorescence when challenged with $\mathrm{CaCl}_{2}$ additions (red arrows), indicating robust MCU-dependent uptake of $\mathrm{Ca}^{2+}$, this response was inhibited in $M c u^{\text {llfl-MyoD-Cre}}$ mitochondria (Figure 1E and Supplemental Figure 1C). The first $2 \mathrm{Ca}^{2+}$ addition points show artifactual signal diminution in the $\mathrm{Mcu}$-deleted mitochondria due to technical limitations of the assay system related to $\mathrm{Ca}^{2+}$ mixing and buffering (data not shown). Together, these results show that acute skeletal muscle mitochondrial $\mathrm{Ca}^{2+}$ influx is inhibited in Mcut/fl-MyoD-Cre mice. Importantly, loss of $\mathrm{Mcu}$ in skeletal muscle did not influence the total intracellular $\mathrm{Ca}^{2+}$ transient amplitude or $\mathrm{SR} \mathrm{Ca}^{2+}$ reuptake rates in isolated FDB myofibers upon $1 \mathrm{~Hz}$ pacing (Supplemental Figure 1, D and E).

Interestingly, loss of $\mathrm{Mcu}$ with MyoD-Cre throughout development did not affect body weight, muscle weights, or fiber cross-sectional areas versus controls at 4 months of age (Figure 1, F-H), nor did it negatively affect skeletal muscle histology or mitochondrial ultrastructure by electron microscopy (Figure 1, I and J, and Supplemental Figure 2). In support of these observations, Western blot analysis of the activation states of Akt and GSK3 $\alpha / \beta$ revealed no alterations in the phosphorylation status of these growth-regulating kinases in the absence of $\mathrm{Mcu}$, further suggesting that MCU does not directly control muscle growth (Supplemental Figure 3).

Deletion of Mcu in adult skeletal muscle inhibits $\mathrm{Ca}^{2+}$ influx but not growth. To investigate the contribution of MCU to adult skeletal muscle growth, we crossed $\mathrm{Mcu}^{\mathrm{fl} / \mathrm{fl}}$ animals with transgenic mice expressing a tamoxifen-inducible Cre recombinase (MerCreMer [MCM]) under the control of the skeletal muscle $\alpha$-actin promoter (Ska-MCM; Figure 2A and ref. 18). Tamoxifen chow was administered to 8-week-old adult mice for 4 weeks to mediate $\mathrm{Mcu}$ deletion, after which mice were analyzed at 28 weeks of age (Figure $2 \mathrm{~B}$ ). This long time point for subsequent analysis was needed because of low turnover of MCU protein from mitochondria in vivo, as we observed previously in the heart (14). Following this dosing regimen, MCU protein levels were reduced by greater than $90 \%$ in $M c u^{f / f-S k a-M C M}$ skeletal muscle mitochondria compared with $M c u^{f / f l}$ controls (Figure 2C). Similar to the Mcut/fl-MyoD-Cre model, Mcut/fl-Ska-MCM muscle mitochondria displayed blunted mitochondrial $\mathrm{Ca}^{2+}$ uptake as compared with control animals (Figure 2E), yet this impairment in 
A

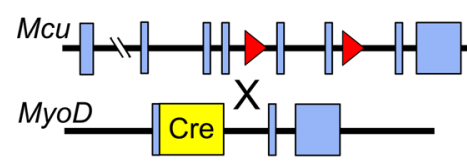

B

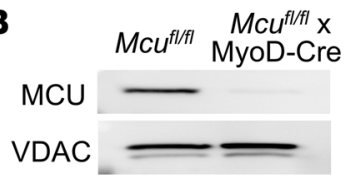

E

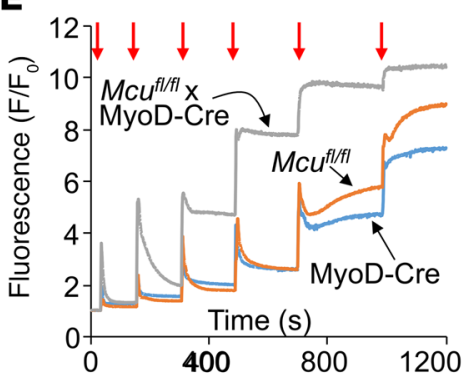

C

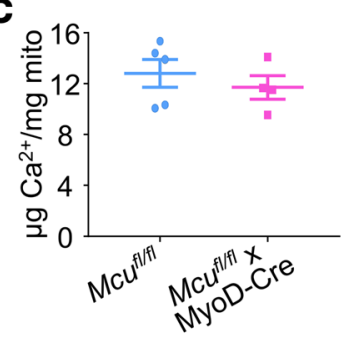

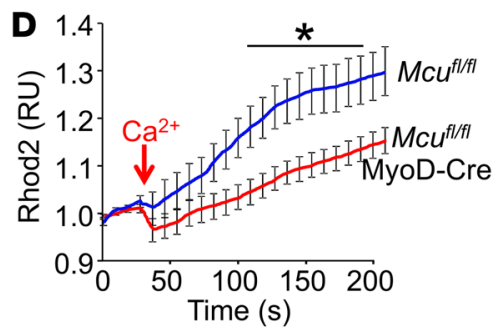
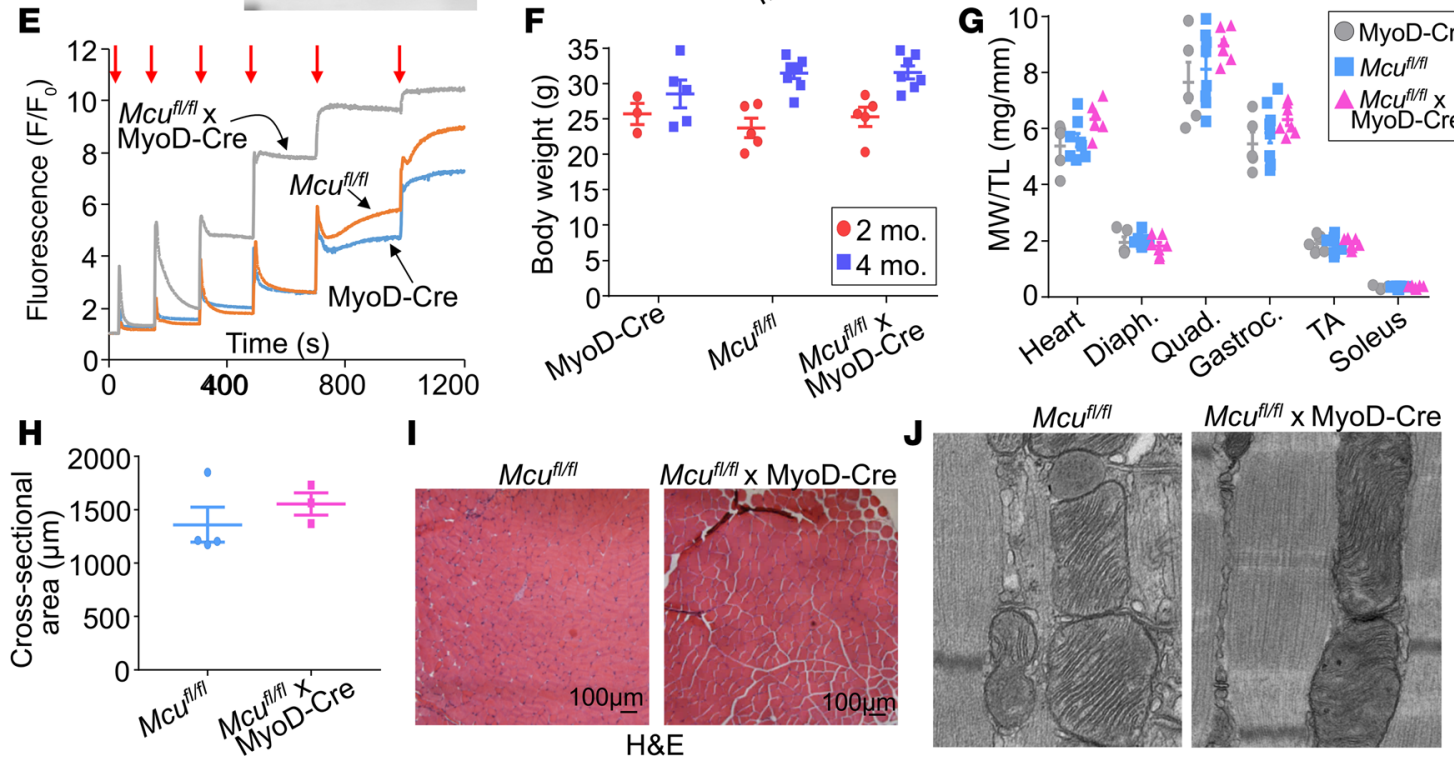

I
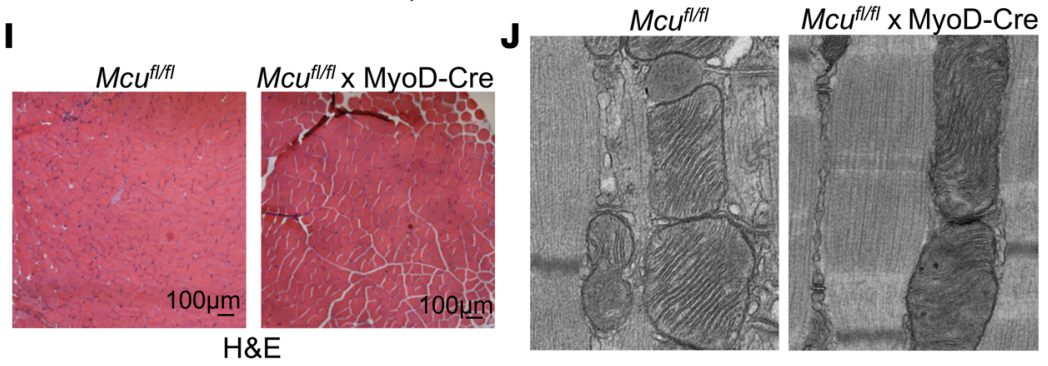

Figure 1. Loss of Mcu during skeletal muscle embryonic development does not cause growth impairment. (A) Strategy to generate the $M c u^{f / f-M y o D-C r e}$ mice. (B) Western blot analysis of MCU expression from muscle mitochondria isolated from 2-month-old animals. The voltage-dependent anion channel (VDAC) was used as the protein loading control. (C) Quantification of total $\mathrm{Ca}^{2+}$ content in isolated muscle mitochondria (mito) from the indicated groups; $n=5\left(\mathrm{Mcu}^{\mathrm{f} / \mathrm{f})}\right), n$ $=4\left(M^{\prime f f / f-M y o D-C r e}\right)$. Student's $t$-test was used to analyze groups for statistical significance. (D) Stimulated Ca ${ }^{2+}$ uptake in saponin permeabilized FDB myofibers challenged with a $20 \mu \mathrm{M} \mathrm{Ca}{ }^{2+}$ bolus (arrow); average axial mitochondrial Rhod-2 fluorescence (relative units, RU) was tracked from the given genotypes.

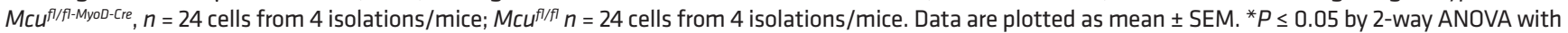
Bonferroni's post hoc test. Average peak data are shown in Supplemental Figure 1B. (E) Mitochondrial $\mathrm{Ca}^{2+}$ uptake from isolated muscle mitochondria of the genotypes shown measured using the Calcium Green- $5 \mathrm{~N}$ uptake assay. Calcium Green-5N signal was a ratio to baseline fluorescence values $\left(F / F_{0}\right)$. Mitochondria were challenged with $10 \mu \mathrm{M} \mathrm{CaCl}_{2}$ (red arrows). (F) Body weights of mice (g) from the indicated groups at 2 and 4 months of age. For animals at 2 months of age,

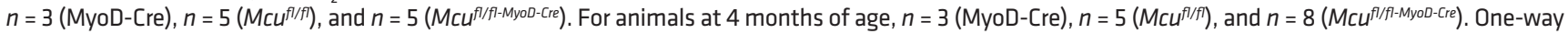
ANOVA with Dunnett's multiple comparisons test was used for statistical analysis. (C) Muscle weight normalized to tibia length (MW/TL) at 4 months of age. Muscles analyzed are shown, and heart weight was normalized to tibia length; $n=5$ (MyoD-Cre), $n=8$ (Mcu f/ff), and $n=8$ (Mcuf/f-Myoo-cre). One-way ANOVA with Dunnett's test was used for statistical analysis. (H) Quantification of myofiber cross-sectional area from 4-month-old animals from the indicated genotypes; $n=3$ per group; Student's 2-tailed $t$-test was used to analyze groups. (I) Transverse H\&E-stained quadriceps histological sections from 4-month-old mice. (J) Representative electron micrographs of quadriceps sections at $\times 4,000$ magnification. Scatter plots show individual values and mean \pm SEM.

mitochondrial $\mathrm{Ca}^{2+}$ influx had no impact on muscle weights and tissue integrity assessed by histology (Figure 2, D and F).

Adeno-associated virus serotype 9 expressing Cre recombinase (AAV9-Cre) was also directly injected into the tibialis anterior (TA) muscle of 6-week-old $\mathrm{Mcu}^{\mathrm{flf} / \mathrm{l}}$ animals as another means of mediating adult muscle-specific $M c u$ deletion, which was compared with AAV9 expressing GFP (AAV9-GFP) (Figure 2G). MCU protein levels were reduced in TA muscle lysates of AAV9-Cre-transduced animals compared with AAV9-GFP controls at 8 weeks following viral transduction (Figure 2H). Yet no changes in TA muscle weight were observed, nor was tissue pathology present (Figure 2, I and J). Collectively, these results suggest that loss of MCU-dependent $\mathrm{Ca}^{2+}$ signaling was not associated with a significant change in skeletal muscle growth or histologically assessed structure.

$M C U \mathrm{Ca}^{2+}$ signaling controls acute mitochondrial energy production. Heart-specific deletion of $\mathrm{Mcu}$ caused a deficit in acute exercise performance in mice, as well as inhibition of $\beta$-adrenergic-stimulated respiration in isolated cardiomyocytes (14). To examine whether MCU plays a similar role in skeletal muscle, respiration rates in isolated muscle mitochondria were first examined under baseline conditions and conditions 
A

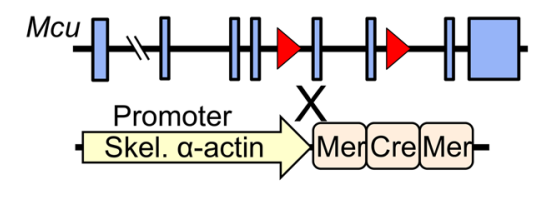

B
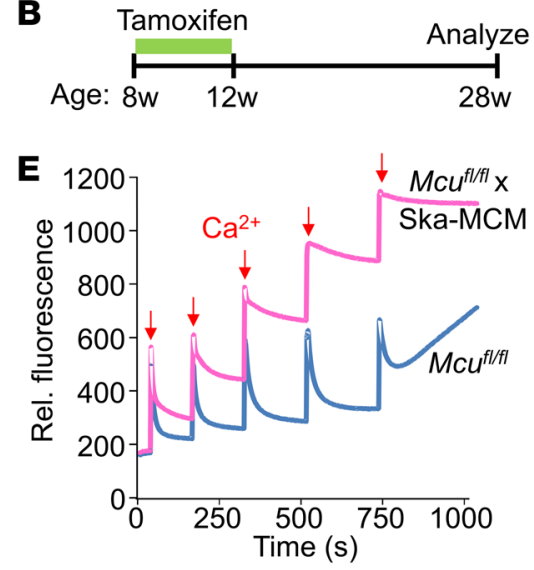

I

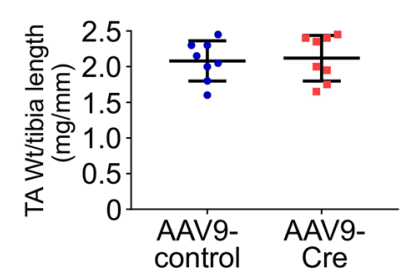

C

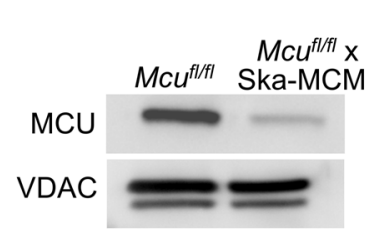

$\mathbf{F}$
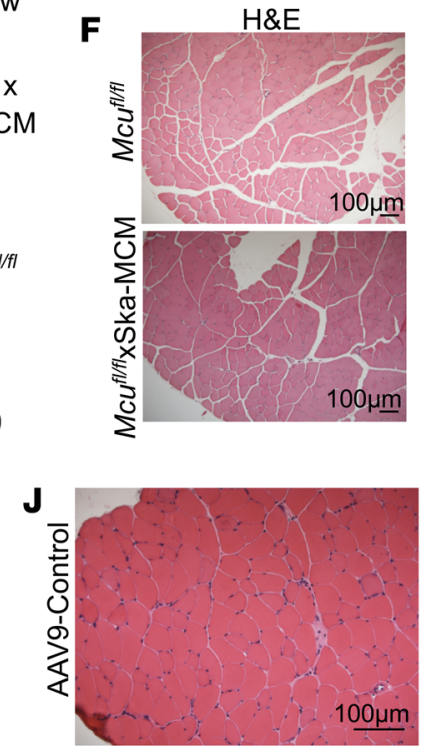

D

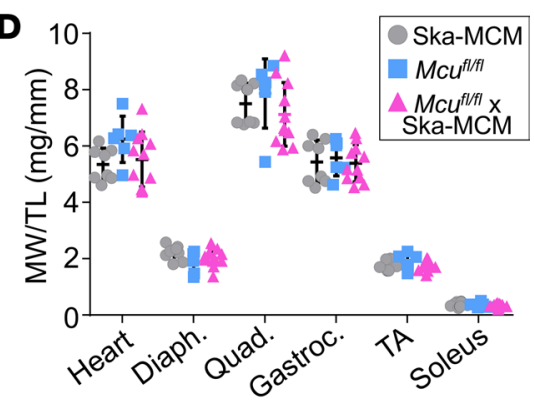

G
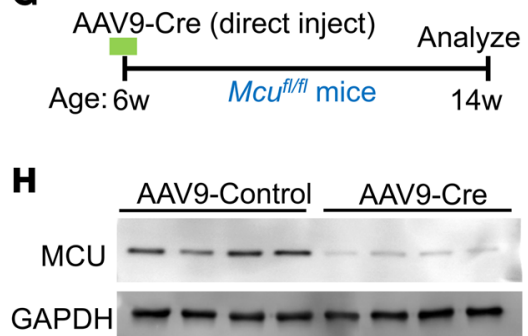

Figure 2. Mitochondrial $\mathrm{Ca}^{2+}$ influx does not regulate postnatal skeletal muscle growth. (A) Strategy for the generation of the $M c u^{f / f-5 k a-M C M}$ mice. Skel, skeletal muscle. (B) Tamoxifen dosing regimen to induce Mcu deletion. Mice were fed tamoxifen chow for 4 weeks $(400 \mathrm{mg} / \mathrm{kg})$ and examined at 28 weeks of age. (C) Western blot analysis of MCU expression from isolated muscle mitochondria prepared at 28 weeks of age from the indicated groups. The VDAC was used as the protein loading control. (D) MW/TL from the indicated groups. Muscles analyzed are shown, and heart weight is also normalized to tibia length. $n=8$ (Ska-MCM), $n=5\left(\mathrm{Mcu}^{f / f}\right)$, and $n=11\left(\mathrm{Mcu}^{f / f-\mathrm{fk} a-M C M}\right)$. One-way ANOVA with Dunnett's multiple comparisons test was used for statistical analysis. (E) Calcium Green-5N assay for mitochondrial $\mathrm{Ca}^{2+}$ uptake in isolated muscle mitochondria. Mitochondria were challenged with $10 \mu \mathrm{M} \mathrm{CaCl}, 2$ pulses (red arrows). Rel, relative. (F) Representative transverse H\&E-stained TA histological muscle sections at $\times 100$ magnification. (C) Schematic for experiments using direct AAV9 TA muscle injections. Mcu f//fl animals were injected with virus at 6 weeks of age and analyzed at 14 weeks of age. (H) Western blot analysis of MCU expression from total TA protein lysates of AAV9-Cre- or AAV9-GFP-transduced Mcuf/ff animals. GAPDH was used as a protein loading control. (I) TA MW/TL from the indicated AAV9 experimental groups; $n=8$ per group. Student's 2-tailed $t$-test was used to analyze groups for statistical significance. (J) Representative H\&E-stained TA muscle sections 8 weeks after AAV9 viral transduction. Scatter plots show individual values and mean \pm SEM.

with $\mathrm{Ca}^{2+}$ stimulation. Oxygen consumption was measured using a Clark-type electrode in skeletal muscle mitochondria isolated from 2- to 3-month-old $\mathrm{Mcu}^{\mathrm{fl/f-MyoD-Cre}}$ and $\mathrm{Mcu^{f/f }}$ control mice. Under baseline conditions using pyruvate and malate as substrates, both $\mathrm{Mcu}$-deleted and control mitochondria displayed similar rates of respiration (Figure 3A). Similarly, maximal carbonyl cyanide 4-(trifluoromethoxy)phenylhydrazone-uncoupled (FCCP-uncoupled) respiration was also unchanged in $M c u^{f / f-M y o D \cdot C r e}$ versus $M c u^{f / f}$ control mitochondria, suggesting that mitochondrial respiratory capacity is not affected by $\mathrm{Mcu}$ deletion (Figure $3 \mathrm{~A})$. However, while control mitochondria displayed a robust increase in oxygen consumption with $\mathrm{Ca}^{2+}$ stimulation, this response was abrogated with $\mathrm{Mcu}$ deletion (Figure 3A).

To examine the physiological consequences of $M c u$ deletion, 2-month-old $M c u^{q / f-M y o D-C r e}, M c u^{f / f}$, and MyoD-Cre animals were subjected to 2 treadmill exercise protocols. In the first protocol, animals were rapidly accelerated from rest $(0 \mathrm{~m} / \mathrm{min})$ to sprint $(20 \mathrm{~m} / \mathrm{min})$ conditions over the course of 2 minutes and then challenged to maintain this speed for 20 minutes (Figure 3B). Under these conditions, Mcut/f-MyoD-cre animals displayed a significantly impaired exercise capacity compared with $M c u^{f / f}$ and MyoD-Cre controls (Figure $3 \mathrm{C}$ ). However, in a prolonged acclimatization running protocol, where animals were allowed to transition from rest to running over 30 minutes and then challenged to complete 20 minutes at maximum speed (Figure 3D), there were no differences in exercise capacity between the $\mathrm{Mcu}$-deleted and control groups (Figure 3E). These results suggest that loss of $\mathrm{Mcu}$ in skeletal muscle impairs acute $\mathrm{Ca}^{2+}$-stimulated mitochondrial 
A

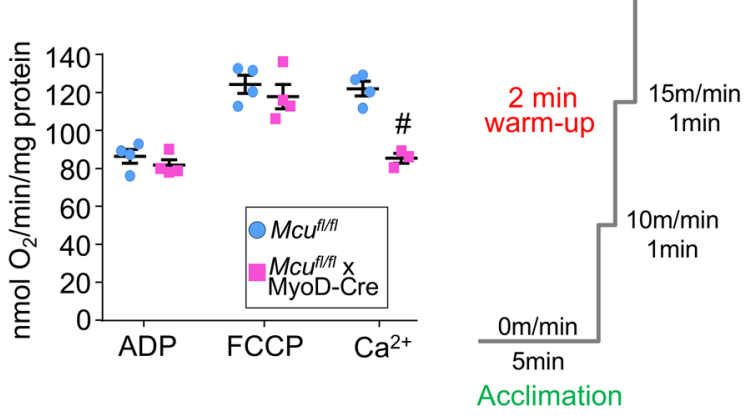

$20 \mathrm{~m} / \mathrm{min}$ SPRINT

$20 \mathrm{~min}$

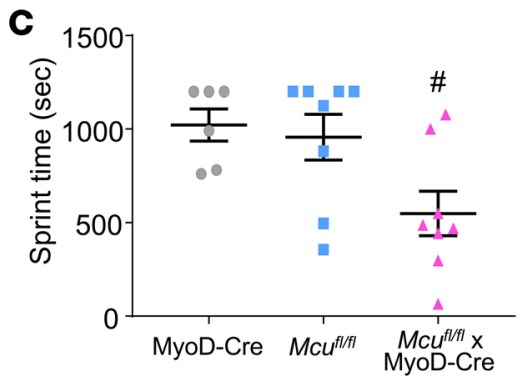

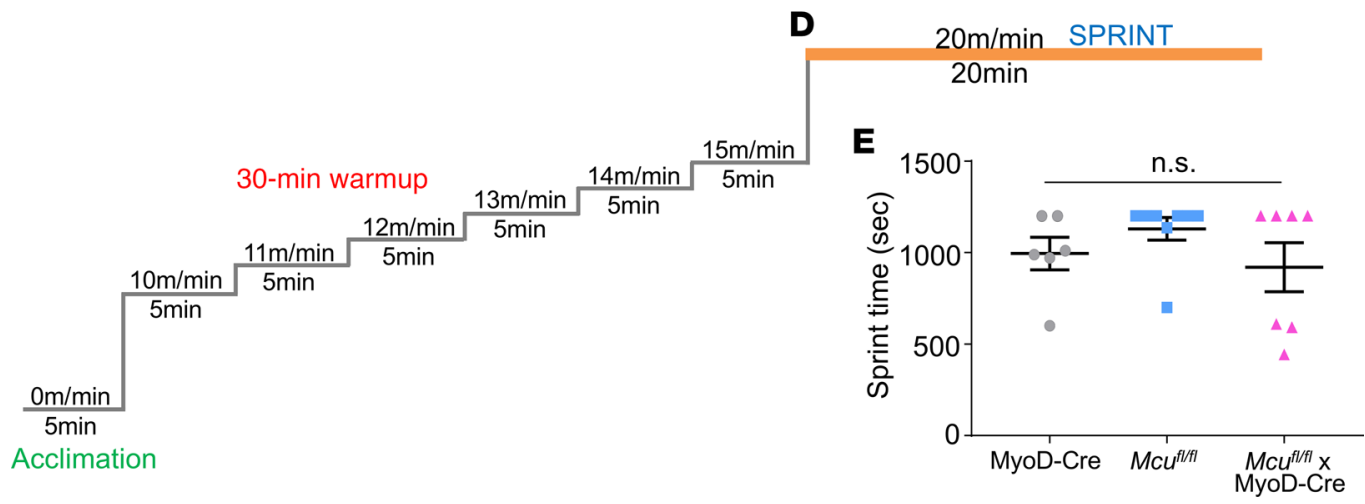

Figure 3. Uniporter-dependent mitochondrial $\mathrm{Ca}^{2+}$ signaling controls rapid upregulation of mitochondrial energy production. (A) State 3 mitochondrial oxygen consumption rate of isolated muscle mitochondria with ADP from the indicated groups of mice at baseline, after stimulation with $5 \mu \mathrm{M} \mathrm{FCCP}$, or after stimulation with $100 \mu \mathrm{M} \mathrm{CaCl}_{2} \cdot n=4$ per group. Student's 2 -tailed $t$-test was used to analyze groups for statistical significance. ${ }^{\#} P<0.05$ versus $\mathrm{Mcu}^{f / / f}$ control. (B) Treadmill regimen for acute sprinting of mice. (C) Quantification of sprint capacity in the indicated groups of mice

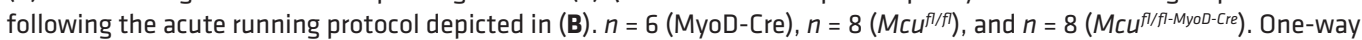
ANOVA with Dunnett's multiple comparisons test was used for statistical analysis. ${ }^{P} P<0.05$ versus $M c u^{f / f l}$ control. (D) The treadmill regimen for the prolonged acclimatization protocol and $(E)$ quantification in the indicated groups follow-

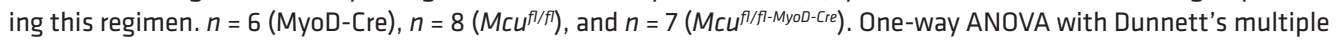
comparisons test was used for statistical analysis. Scatter plots show individual values and mean \pm SEM.

energy upregulation but that with time this acute $\mathrm{Ca}^{2+}$-stimulated process is compensated by either other $\mathrm{Ca}^{2+}$ influx pathways or other metabolic compensations.

Finally, metabolomics analysis of quadriceps muscle of $M c u^{A / f-M y o D-C r e}$ animals as compared with $M c u^{f / f l}$ controls under exercise conditions revealed a significant increase in tissue lactate, supporting impaired glucose oxidation. Citrate, glutamine, glycine, valine, and phenylalanine levels were also elevated, suggesting alterations in substrate entry into the TCA cycle with associated changes in metabolite flux (Supplemental Table), which may underlie the exercise phenotype observed in Mcu-deficient animals (Figure 3C).

Mcu deletion in skeletal muscle augments fatty acid oxidation. To further evaluate how loss of Mcu affected skeletal muscle metabolism and functional performance, we analyzed contractile fatigue properties of TA muscle in a whole leg immobilization preparation from 3-month-old $M c u^{f / f-M y o D-C r e}$ and $M c u^{f / f}$ control animals. Animals were subjected to a muscle fatigue regimen induced by 3 successive cycles of $150-\mathrm{Hz}$ stimulation over 45 seconds. The maximal specific force of a single twitch elicited at $150 \mathrm{~Hz}$ was measured following the completion of each cycle. Surprisingly, $M c u^{f / f-M y o D-C r e}$ TA muscle displayed significantly less fatigue and maintained greater force production compared with the $M c u^{q / f}$ controls (Figure 4A).

This fatigue profile suggested that loss of $\mathrm{Mcu}$ might enhance functional recovery in mice after exercise, possibly due to altered metabolic substrate usage. Indirect calorimetry was used in whole mice to calculate the respiratory exchange ratio (RER) to infer metabolic substrate usage. RER values of 1 indicate preferential glucose oxidation, while RER values of 0.7 indicate preferential fatty acid oxidation, which for skeletal muscle is characteristic of recovery. Under basal resting conditions, the RER was unchanged in both ad libitum fed and fasting $M c u^{f / f-M y o D-C r e}$, MyoD-Cre, and $M c u^{\nexists / f}$ mice (Figure 4, B and C). However, examination of 
A
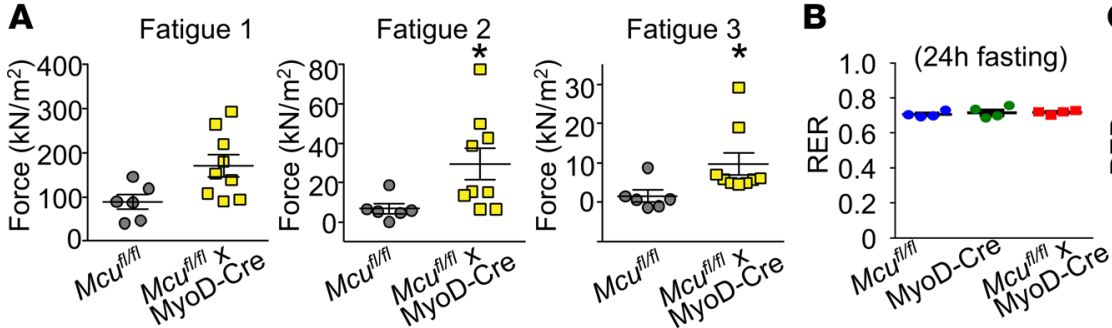

C

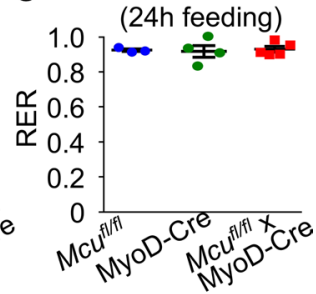

D
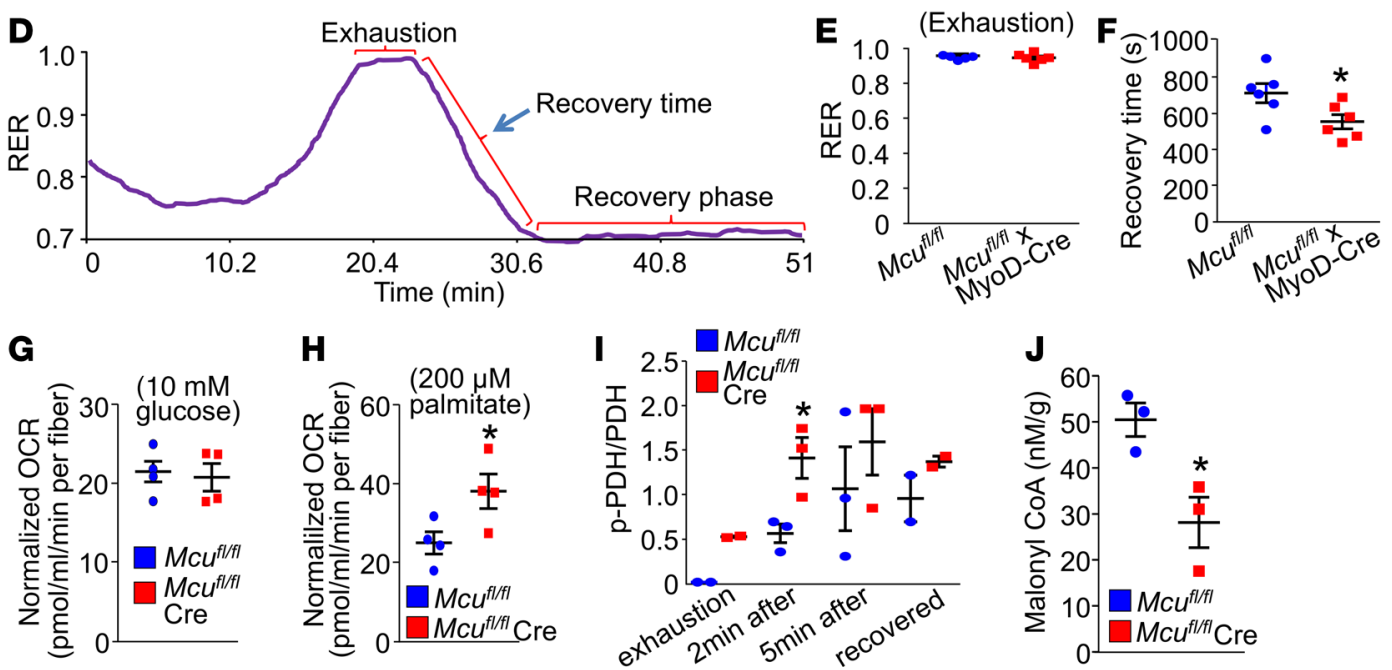

Figure 4. Mcu deletion in skeletal muscle causes a metabolic shift toward fatty acid oxidation. (A) Quantification of TA muscle-specific force following multiple rounds of fatigue in the indicated genotypes of mice. $n=6\left(M^{\prime} u^{f / f}\right), n=9$ (Mcu f/f-MyoD-cre $)$. Student's 2-tailed $t$-test was used to analyze groups for statistical significance. ${ }^{*} P<0.05$ versus $\mathrm{Mcu}^{\mathrm{fl} / \mathrm{fl}}$ control. kN, kilonewton. (B) Average RER values from 2-month-old mice of the indicated genotypes following 24 hours of fasting or (C) 24 hours of feeding. $n=4$ per group. (D) Representative graph tracing of RER measurements taken throughout the rest-exhaustion-recovery experiment for mice subjected to treadmill running in metabolic cages. Exhaustion, recovery time, and recovery phases are indicated. (E) Quantification of average RER values at exhaustion and (F) during recovery time in the indicated groups. $n=8$ per group. (G) $\mathrm{O}_{2}$ consumption rate (OCR) of isolated FDB myofibers when glucose was given as a metabolic substrate or $(\mathbf{H})$ palmitate was given at the shown concentrations. The myofibers were isolated from $n=4\left(M_{\left.c u^{f / f}\right)}\right), n=4\left(M_{\left.c u^{f / f-M y o D-C r e}\right)}\right.$ mice. (I) Western blot analysis-based quantification of phosphorylated PDHE1 $\alpha$ (p-PDHE1 $\left.\alpha\right)$ and total PDH in gastrocnemius muscle harvested at exhaustion ( $n=2$ per group), 2 minutes after exhaustion ( $n=3$ per group), 5 minutes after exhaustion ( $n=3$ per group), and during recovery ( $n=2$ per group). (J) Malonyl-CoA levels measured by ELISA from quadriceps taken from mice 2 minutes after exhaustion. $n=$ 3 per group. Student's 2-tailed $t$-test was used for statistical analysis. Scatter plots show individual values and mean $\pm S E M .{ }^{*} P<0.05$ versus Mcufl/fl control.

RER values over the course of a 51-minute exercise protocol uncovered a change in $M c u^{q / f-M y o D-c r e}$ versus the $M c u^{q / f l}$ control mice. The data show that both $M c u^{q / f-M y o D-C r e}$ and $M c u^{l / f l}$ mice display similar times to exhaustion and RER values at exhaustion, and both groups fully utilized glucose at exhaustion (Figure 4, D and E). However, when the run-to-exhaustion phase was stopped, the $M c u^{\text {f/f-MyoD-Cre }}$ animals showed faster recovery with a more rapid shift toward fatty acid oxidation compared with $M c u^{f / f}$ control mice, as evidenced by a faster decline in RER values toward 0.7 (Figure 4, D and F). This faster time to recovery of the Mcut/ff-MyoD.Cre animals suggests that $\mathrm{Mcu}$ deletion enhanced fatty acid oxidation that is unmasked with fatigue.

To more specifically examine the role of MCU in affecting metabolic substrate usage of skeletal muscle, $\mathrm{O}_{2}$ consumption (respiration) was examined from intact FDB myofibers isolated from 2-month-old $M c u^{f / f l}$ and $M c u^{f / f-M y o D-C r e}$ mice. While respiration was unchanged with $M c u$ deletion when glucose was used as a substrate (Figure 4G), Mcu ${ }^{A / f-M y o D-C r e}$ myofibers displayed significantly increased rates of palmitate-supported respiration as compared with $M c u^{\ell / f l}$ controls (Figure $4 \mathrm{H}$ ). These results suggest that deletion of $M c u$ enhances $\beta$-oxidation in skeletal muscle.

Consistent with an Mcu deletion-induced metabolic shift toward fatty acid oxidation, Western blot analysis of the phosphorylation status of the pyruvate dehydrogenase (PDH) complex was performed. $\mathrm{PDH}$ is a key enzyme regulating entry of glucose-derived metabolites into mitochondrial oxidative phosphorylation. Phosphorylation of the PDH complex subunit PDH1E $\alpha$ was significantly increased, and $\mathrm{PDH}$ activity was commensurately decreased in $M c u^{\text {Af/-MyoD-Cre }}$ total quadriceps protein lysates at baseline compared with MyoD-Cre and $M c u^{f / f}$ control mice (Supplemental Figure 4, A-C). We also analyzed mice 
after exhaustion and recovery from running for $\mathrm{PDH}$ phosphorylation, which again was greater in quadriceps lysates from $M c u^{f l / f-M y o D-C r e}$ mice at exhaustion and during recovery compared with $M c u^{f l f l}$ control mice (Figure 4I). These results suggest a partial inhibition of baseline and exercise-induced glucose oxidation. Importantly, malonyl-CoA, an inhibitor of carnitine palmitoyltransferase 1, a key enzyme regulating fatty acid entry into the $\beta$-oxidation pathway, was significantly reduced in $M c u^{f / f-M y o D-C r e}$ exercised muscle as compared with controls with exercise but not at baseline, further supporting preferential fatty acid metabolism in Mcu-deficient muscle (Figure 4J and Supplemental Figure 4D). No differences in total muscle glycogen were observed (Supplemental Figure 4E).

Finally, while body weights were unchanged in $M c u^{f l f-M y o D-C r e}$ versus $M c u^{f l f l}$ controls as they aged to 7 months, $M c u$ deletion was associated with a significant decrease in total fat mass as measured by magnetic resonance imaging (Supplemental Figure 5, A-C), suggesting that MCU regulation of muscle metabolic substrate selection affects the total metabolic profile of these mice and likely greater total energy usage. Because fat mass is a relatively minor component of the total animal weight, total body mass was not significantly different between the 2 groups, despite the mild but significant reduction in fat mass with aging.

\section{Discussion}

Mitochondrial $\mathrm{Ca}^{2+}$ signaling serves as an important link between contraction of the heart and energetics because acute $\mathrm{Ca}^{2+}$ entry through the $\mathrm{MCU}$ confers fast, dynamic regulation of mitochondrial energy output $(14,15)$. Similar to the heart, the MCU in skeletal muscle also mediates mitochondrial $\mathrm{Ca}^{2+}$ transport, which, by using an shRNA approach in mice or in a fully germline deficient mouse model, was suggested to influence muscle function and growth $(13,16)$. Here, we used 3 muscle-specific genetic approaches in the mouse to specifically address the functional role of MCU, which is a critical consideration because fully germline deficient $\mathrm{Mcu}^{-/-}$mice are known to have compensatory changes that mask some functions of $\mathrm{MCU}(13,16)$. We demonstrated that skeletal muscle-specific deletion of $\mathrm{Mcu}$ during development or adulthood each resulted in inhibited acute mitochondrial $\mathrm{Ca}^{2+}$ uptake. Contrary to a previous report where viral-mediated shRNA knock down of MCU in skeletal muscle caused muscle atrophy (16), our study revealed that genetic ablation of $M c u$ at 3 stages of development (fetal, postnatal, and adult) had no significant impact on overall muscle growth. However, we observed that MCU and mitochondrial $\mathrm{Ca}^{2+}$ are intimately linked to muscle metabolism because $\mathrm{Mcu}$ deletion caused an impairment in mitochondrial energy production stimulated by $\mathrm{Ca}^{2+}$, alterations in TCA cycle substrate flux, and a shift toward fatty acid metabolism that ultimately resulted in a mild but significant reduction of total body fat mass with aging.

Skeletal muscle is a metabolic omnivore with the capacity to use both glucose and fatty acids as fuel sources (19). It has long been known that preferential usage of glucose versus fatty acids can be influenced by a number of factors, including energetic demand (exercise) and nutrient availability (obesity and diabetes) (20). In this study, we observed that deletion of $M c u$ specifically in skeletal muscle inhibited acute mitochondrial $\mathrm{Ca}^{2+}$ influx and resulted in 2 seemingly incongruent physiological phenotypes. Animals with skeletal muscle-specific deletion of $M c u$ displayed a deficit in running capacity in a sprinting protocol, suggesting impaired muscle function (Figure 3C), yet these animals displayed enhanced muscle function and metabolic recovery during fatigue (Figure 4, A and F). Indirect calorimetry during this run-to-exhaustion and recovery regimen revealed 2 phases of substrate usage. During the run-to-exhaustion phase, mice displayed an increased reliance on glucose metabolism, whereas during the recovery phase, fatty acid metabolism predominated (Figure 4D) and mice with muscle-specific deletion of $M c u$ recovered more quickly (Figure 4F). Analysis of oxygen consumption in isolated muscle fibers revealed that loss of $M c u$ led to an enhanced ability to respire when fatty acids were used as substrates. Similarly, alterations in PDH phosphorylation and malonyl-CoA levels were consistent with a shift toward fatty acid oxidation when $M c u$ is deleted from muscle. However, to truly prove that skeletal muscle from $\mathrm{Mcu}$-deficient mice preferentially uses fatty acids in vivo under working conditions, NMR spectroscopy would be needed.

The loss of MCU produced a compensatory upregulation in fatty acid usage, as shown by a number of direct and indirect assays in Figure 4. For example, acute maximal exercise of skeletal muscle in mammals typically involves the usage of all possible metabolic pathways and substrates, both mitochondrial-dependent and -independent, and a defect in any of these components would likely compromise maximal performance. The acute uptake of $\mathrm{Ca}^{2+}$ into mitochondria in the immediate phase of exercise stimulates TCA cycle dehydrogenases, the electron transport chain, and ATP synthase to rapidly augment high-energy phosphate production (21). 
Under these acute conditions, Mcu-deleted mice showed a significant defect in performance on the treadmill, presumably because they were not able to rapidly augment mitochondrial fatty acid and glucose oxidation, yet glycolysis and lactate production, which are major aspects of anaerobic sprinting, still provide much of the capacity. Indeed, the increased lactate seen after exercise in the Mcu-null mice (Supplemental Table) may be evidence of reduced glucose oxidation during exercise that would normally consume glycolysis-derived pyruvate/lactate. However, warming up the mice for 30 minutes at lower speeds allows the mitochondria in skeletal muscle to presumably still accumulate $\mathrm{Ca}^{2+}$ through other uptake processes, as we showed previously with $\mathrm{Mcu}$ deletion from the heart (14). Because of unknown compensatory augmentations in skeletal muscle metabolism in the absence of $\mathrm{MCU}$, gradual mitochondrial $\mathrm{Ca}^{2+}$ loading through alternative pathways now permitted the mice to sustain a more robust exercise regimen thereafter (Figure $3 \mathrm{E}$ ) and even deal more effectively with fatigue (Figure 4A). We hypothesize that MCU functions as an evolutionary adaptive gene that underlies rapid induction of metabolic output to presumably allow for reduced baseline homeostatic energy usage. In its absence there appears to be a compensation that permits greater fatty acid usage, maybe because this substrate is less reliant on mitochondrial $\mathrm{Ca}^{2+}$ load compared with glucose oxidation, which is regulated by PDH activity, a direct target of mitochondrial $\mathrm{Ca}^{2+}$ signaling. Also, in the absence of MCU there appears to be less mitochondrial glucose oxidation or increased glycolysis, although total glucose usage is unaffected (Figure 4G), contributing to the observed increase in lactate, as seen in other systems (22). However, to really know these relationships with certainty, in vivo substrate usage profiling with NMR spectroscopy would be needed at rest, during sprinting, and with sustained exercise.

In summary, we conclude that deletion of $M c u$ in skeletal muscle causes a loss in metabolic flexibility with an enhanced reliance on fatty acid metabolism, which is unmasked under conditions of prolonged stress and aging. Because skeletal muscle is the largest organ in the body with the greatest impact on whole body energy expenditure, this work also highlights a new role for MCU in the control of systemic metabolism.

\section{Methods}

Animals. $M c u^{f l f f}$ gene-targeted mice were generated previously (14). To generate the $M c u^{f / f-M y o D-C r e}$ animals, $M c u^{f / f l}$ and MyoD-Cre knockin mice expressing Cre recombinase under the control of the Myod gene locus (17) were crossed. To generate the $M c u^{f / f-S k a-M C M}$ animals, $M c u^{f / f l}$ mice were crossed with transgenic mice in which the human skeletal $\alpha$-actin promoter (18) drives expression of the MCM CDNA, which is a tamoxifen-inducible Cre recombinase. $M c u$ deletion in the $M c u^{\text {llff-Ska-MCM }}$ animals was induced by feeding 8-week-old mice tamoxifen citrate mouse chow ( $400 \mathrm{mg} / \mathrm{kg}$; Harlan Laboratories) for 4 weeks. All animal experiments were approved and performed in accordance with Cincinnati Children's Hospital Medical Center's and Emory University's IACUCs. Mice were randomly assigned to experimental groups, although because they were genetically identical for each of the groups and matched for age and sex ratio, randomization is a rather unimportant consideration. Both male and female mice were used in an equal ratio, and no sex-specific differences were observed. Experiments with mice were performed in a blinded manner where possible.

Adeno-associated viruses and direct muscle injections. AAV9-Cre and AAV9-GFP were purchased from Vigene Biosciences. The TA muscle of 8-week-old $M c u^{f / f t}$ animals was injected with $50 \mu 1$ sterile PBS containing $1 \times 10^{12}$ viral particles using a 30.5-gauge needle. Muscle was harvested 8 weeks following viral transduction. The muscle from one leg was flash frozen for immunoblotting, and the muscle from the other leg was processed for histology.

Histological analyses. Muscles were harvested, fixed in 10\% formalin, and embedded in paraffin. Then, $5-\mu \mathrm{m}$ tissue sections were prepared and stained with H\&E. The myofiber cross-sectional area was measured using NIH Image J software. For transmission electron microscopy, quadriceps muscle was harvested, fixed in $3.5 \%$ glutaraldehyde and $0.15 \%$ sucrose in $0.1 \mathrm{M}$ sodium cacodylate, $\mathrm{pH} 7.4$, postfixed in $1 \% \mathrm{OsO}_{4}$, dehydrated, and embedded in epoxy resin, as previously described (23). Imaging was performed using a Hitachi 7600 electron microscope.

Mitochondrial isolation and $\mathrm{Ca}^{2+}$ measurements. Calcium uptake in isolated skeletal muscle mitochondria was performed using a Calcium Green-5N assay (Molecular Probes) (14). Skeletal muscle mitochondria were isolated by differential centrifugation in MS-EGTA buffer (225 mM mannitol, $75 \mathrm{mM}$ sucrose, $5 \mathrm{mM}$ HEPES, and 1 mM EGTA, pH 7.4), and the $\mathrm{Ca}^{2+}$ uptake assay was conducted as previously described (14). Briefly, mitochondria were suspended in buffer containing $125 \mathrm{mM} \mathrm{KCl}, 20 \mathrm{mM}$ HEPES, $2 \mathrm{mM} \mathrm{MgCl}{ }_{2}$, $2 \mathrm{mM}$ potassium phosphate, $40 \mu \mathrm{M}$ EGTA (pH 7.2), $500 \mu \mathrm{M}$ Calcium Green-5N (Molecular Probes), 7 $\mathrm{mM}$ pyruvate, and $1 \mathrm{mM}$ malate. Mitochondria were challenged with additions of $10 \mu \mathrm{M} \mathrm{CaCl}_{2}$. Calcium Green-5N fluorescence was monitored using a Synergy 2 microplate reader (BioTek). 
For mitochondrial $\mathrm{Ca}^{2+}$ measurements in intact myofibers, FDB muscle fibers were isolated as described previously (24). For baseline and permeabilized mitochondrial $\mathrm{Ca}^{2+}$ measurements, isolated FDB fibers were incubated with $5 \mu \mathrm{M}$ Rhod-2 AM (Thermo Fisher Scientific) for 60 minutes at room temperature (25). Fibers were treated with Rhod-2 AM in suspension for 45 minutes before they were attached to glass-bottom culture dishes (MatTek Corporation) precoated with laminin (Thermo Fisher Scientific) and incubated for an additional 15 minutes. Fibers were then given a 30-minute room temperature de-esterification wash to remove unincorporated dye. For baseline mitochondrial $\mathrm{Ca}^{2+}$ measurements, myofibers were then imaged for Rhod-2 fluorescence on a Nikon A1R LUN-V inverted confocal microscope (561 nm excitation $/ 600 \mathrm{~nm}$ detection $/ \times 60$ objective) at room temperature $\left(22^{\circ} \mathrm{C}-24^{\circ} \mathrm{C}\right)$. Analysis was conducted using Nikon Elements software. Individual groups of axial mitochondria were manually gated to avoid off-target XY fluorescence, and mean baseline Rhod-2 intensity was calculated by averaging 5 mitochondrial regions of interest per myofiber. For permeabilization, washed myofibers were incubated with medium containing $50 \mu \mathrm{g} / \mathrm{ml}$ saponin (Fluka) for 30 minutes at room temperature. Saponin was then washed out and the permeabilized myofibers were incubated in supplemented intracellular buffer (26). Permeabilized myofibers were then imaged as above in a time-lapse experiment and challenged with the single addition of buffer containing $20 \mu \mathrm{M} \mathrm{CaCl}_{2}$. Five to 10 axial mitochondria regions of interest, as described above, were used to calculate average myofiber Rhod-2 intensity throughout the time lapse. Rhod-2 fluorescence values were normalized to initial fluorescence values for stimulated $\mathrm{Ca}^{2+}$ uptake experiments.

For measurements of cytosolic $\mathrm{Ca}^{2+}$ transients in intact FDB myofibers, fibers were loaded with $10 \mu \mathrm{M}$ Fluo-4 AM (Molecular Probes), for 20 minutes followed by 20 minutes of dye de-esterification in $\mathrm{Ca}^{2+}$-free Tyrode's solution. $\mathrm{Ca}^{2+}$ transient measurements were acquired from FDB fibers bathed in Tyrode's solution containing $2 \mathrm{mM} \mathrm{CaCl}_{2}$. Electrical field stimulation was applied using an IonOptix MyoPacer Cell Stimulator $(1 \mathrm{~Hz}, 10 \mathrm{~ms}$ duration, $32 \mathrm{~V}$ ), and Fluo-4 was imaged by laser scanning confocal microscopy (Olympus FV1000, $488 \mathrm{~nm}$ excitation and $515 \pm 15 \mathrm{~nm}$ emission). All experiments were performed at room temperature, and data were analyzed using Olympus FV1000 FluoView microscope software and Clampfit.

Mitochondrial respiration. Oxygen consumption was measured on isolated skeletal muscle mitochondria using an Oxygraph Plus instrument (Hansatech) as described previously (13). Mitochondrial oxygen consumption was measured in respiration buffer containing $120 \mathrm{mM} \mathrm{KCl}, 5 \mathrm{mM}$ MOPS, $0.1 \mathrm{mM}$ EGTA, 5 $\mathrm{mM} \mathrm{KH}_{2} \mathrm{PO}_{4}, 0.2 \%$ BSA, $10 \mathrm{mM}$ glutamate, and $2 \mathrm{mM}$ malate. ADP-stimulated respiration was initiated with $0.5 \mathrm{mM}$ ADP, uncoupled respiration was initiated with $5 \mu \mathrm{M} \mathrm{FCCP}$, and $\mathrm{Ca}^{2+}$-stimulated respiration was initiated with $0.1 \mathrm{mM} \mathrm{CaCl}_{2}$.

Oxygen consumption was also measured in freshly isolated myofibers from FDB muscles. For this procedure FDB muscle was first incubated in $5 \mathrm{~mL}$ DMEM (GE Healthcare Lifescience) containing $0.2 \%$ collagenase type I (Sigma-Aldrich), $1 \mathrm{mM}$ sodium pyruvate (Sigma-Aldrich), and 1\% penicillin/streptomycin (Thermo Fisher Scientific) in a 35-mm cell culture dish (Denville) (27). Muscles were placed at $37^{\circ} \mathrm{C}$ in an incubator with $5 \% \mathrm{CO}_{2}$ (Thermo Fisher Scientific) and digested for 75 to 90 minutes. Then the muscles were triturated using large-bore glass pipettes under the Olympus SZH10 stereo microscope until individual fibers were visible in the dish. The isolated fibers were washed several times in $60-\mathrm{mm}$ cell culture dishes (Denville) containing DMEM supplemented with 20\% FBS (VWR Life Science) and 10\% horse serum (Gibco). FDB fibers were then placed in horse serum-precoated, 12-well cell culture dishes (Denville) and incubated at $37^{\circ} \mathrm{C}$ in an incubator with $5 \% \mathrm{CO}_{2}$ (Thermo Fisher Scientific) for 30 minutes. Culturing medium was changed to assay medium (28) containing either $10 \mathrm{mM}$ glucose or $200 \mu \mathrm{M}$ BSA-conjugated palmitate (Sigma-Aldrich) and incubated at $37^{\circ} \mathrm{C}$ with $5 \% \mathrm{CO}_{2}$ (Thermo Fisher Scientific) for 2 hours. Fiber number per well was counted, and oxygen consumption rate was measured using an Oxygraph Plus instrument (Hansatech). Oxygen consumption rate was normalized to fiber number.

Western blot analysis. Total muscle protein extracts were prepared from quadriceps and TA muscles by homogenization in radioimmunoprecipitation assay (RIPA) buffer supplemented with protease inhibitors (Roche) and phosphatase inhibitors (Calbiochem). Mitochondrial protein extracts were prepared by solubilizing isolated muscle mitochondria with the same RIPA buffer. Proteins were resolved on SDS PAGE gels, transferred to polyvinylidene fluoride (PVDF) membranes (MilliporeSigma), and immunodetected with antibodies. Antibodies used in this study are MCU (Cell Signaling Technology, \#14997; 1:1,000), VDAC/Porin (Abcam; Ab14734; 1:1,000), GAPDH (Fitzgerald, 10R-G109A; 1:5,000), p-PDHE1 $\alpha$ (Ser293) (Novus Biologicals, NB110-93479; 1:500), PDHE1 $\alpha$ (Abcam, Ab110330; 1:500), p-Akt (Ser473) 
(Cell Signaling Technology, \#4051S; 1:1,000), Akt (Cell Signaling, \#9272S; 1:1,000), p-GSK3 $\alpha / \beta$ (Cell Signaling Technology, \#8566S; 1:1,000), and GSK3 $\alpha / \beta$ (Cell Signaling Technology, \#5676; 1:1,000).

Muscle functional analyses and treadmill running. For muscle fatigue experiments, mice were anesthetized with an intraperitoneal injection of pentobarbital, a midline incision running from the ankle to the thigh was created, and the skin and fascia were gently removed to expose the TA muscle. The mouse was then placed in a supine position on the muscle testing apparatus, and the leg was immobilized by securing it with thumbscrews set at the distal femur in a custom rig (Aurora Scientific). The distal muscle tendon junction was tied to a small plastic ring, and the distal tendon was resected and the TA gently sliced off the tibia and secured to a servomotor (Aurora Scientific, 305C) using a plastic ring. Two intramuscular electrodes were placed on either side of the peroneal nerve, and stimulation voltages and optimal muscle length $\left(\mathrm{L}_{0}\right)$ were determined and then adjusted to produce maximal isometric force $\left(\mathrm{P}_{0}\right)$ at a stimulation frequency of 150 $\mathrm{Hz}$. Five consecutive isometric contractions were averaged as a measure of the maximal specific tension. A 2 -minute rest period was taken between contractions. Once $\mathrm{P}_{0}$ was determined, the TA was subjected to a fatigue protocol in which the muscle experienced 3 bouts of isometric tetanic contractions at a stimulation frequency of $150 \mathrm{~Hz}$ and maintained for 45 seconds followed by a single twitch. A 2-minute rest period occurred between every fatigue protocol, and force values were normalized to the muscle's physiological cross-sectional area. Metrics of maximal specific force and relaxation kinetics were determined using MATLAB analysis of the raw force-time transients. For the acute and prolonged acclimatization running protocols, enforced running was performed using an Omni-Pacer LC4/M treadmill (Columbus Instruments International), as detailed in Figure 3.

Indirect calorimetry and treadmill running. Metabolic rates were measured at rest and during exercise as described previously (29). Measurements were taken in metabolic chambers at $24^{\circ} \mathrm{C}$ with or without food but with free access to water. The resting oxygen consumption and carbon dioxide production rates $\left(\mathrm{VO}_{2}\right.$ and $\mathrm{VCO}_{2}$, respectively) were measured every 8 minutes using the Oxymax Plus system (Columbus Instruments International) for 24 hours. Fresh air was delivered into chambers with an electric pump. To monitor energy expenditure during exercise, a sealed motorized treadmill was used. The treadmill had adjustable speed and inclination and was equipped with a small electric shock-delivering grid that was used in the training regimens so that mice were used to the running protocol. Electric shock intensity was set to $1 \mathrm{~mA}$ and inclination was set to $5 \%$. Gas samples from the treadmill chamber were collected every 15 seconds and analyzed by the Oxymax Plus system for measurement of $\mathrm{VO}_{2}$ and $\mathrm{VCO}_{2}$. For the actual experiment, mice experienced acclimation at $3 \mathrm{~m} / \mathrm{min}$ for 15 minutes and then moved over the next 7 minutes to $50 \mathrm{~m} / \mathrm{min}$ and maintained this speed for 3 minutes as full exertion, followed thereafter by 0 $\mathrm{m} / \mathrm{min}$ for 25 minutes as recovery. For the data shown in Figure 4, D-F, conditions were such that all genotypes of mice ran the same exact same time and distance in the protocol, and because body weights were similar, each group of mice performed the same total work. RER was calculated as $\mathrm{VO}_{2} / \mathrm{VCO}_{2}$ with CLAMS data eXamination Tool (Columbus Instruments International).

Metabolic profiling and malonyl-CoA measurements. For skeletal muscle metabolomics profiling, 2-monthold mice were run to exhaustion, quadriceps muscle was harvested 2 minutes after exhaustion, and muscles were snap-frozen in liquid nitrogen. Metabolite extraction, acquisition of one-dimensional ${ }^{1} \mathrm{H}$ NMR spectra using a Bruker Avance II $600 \mathrm{MHz}$ spectrometer, and data analyses were conducted by the Cincinnati Children's Hospital NMR-based Metabolomics Core. Briefly, muscles were homogenized and incubated with methanol/chloroform/water mix to obtain polar metabolites. Vacuum centrifugation was performed to achieve dried metabolites, which were resuspended for NMR spectroscopy acquisition. To measure malonyl-CoA, 2-month-old mice were run to exhaustion and quadriceps harvested and snap-frozen. Additionally, quadriceps from 2-month-old mice at baseline were collected. Muscles were homogenized in ice-cold PBS and subjected to 2 freeze-thaw cycles, and cleared lysates were prepared by centrifugation. Malonyl-CoA levels were subsequently measured using a malonyl-CoA ELISA Kit (MyBioSource).

MRI body composition analysis. Fat mass and lean mass of mice were measured with EchoMRI body composition analyzer from EchoMRI. The mouse was placed in a special plastic holder with restricted movement, and the holder was inserted in the magnet for MRI measurement. Duplicate measurements were performed for each animal during 1 run. Mice were examined every month starting at 1 month of age.

PDH activity assay. PDH activity was measured with a $\mathrm{PDH}$ combo microplate assay kit (Abcam). Quadriceps from mice at 2 to 3 months of age were collected and frozen in liquid nitrogen. Sample preparations as well as measurements were performed as outlined in the kit instructions. 
Muscle glycogen measurement. Muscle glycogen content was measured using the Glycogen Assay Kit (Sigma-Aldrich). TA muscles were collected from mice at 2 to 3 months of age and frozen in liquid nitrogen for tissue processing. Muscles were homogenized in deionized water on ice. Homogenates were boiled for 5 minutes and centrifuged at 13,000 $\mathrm{g}$ for 5 minutes. Supernatants were used for glycogen measurements, and samples were measured according to the product protocol.

Statistics. All results are presented as mean \pm SEM or SD. Statistical significance between 2 groups was determined by 2 -tailed Student's $t$-test and $P<0.05$ was considered significant. One-way ANOVA followed by Dunnett's multiple comparisons test were used to determine statistical significance among 3 groups and $P<0.05$ was considered significant. For stimulated mitochondrial $\mathrm{Ca}^{2+}$ uptake in permeabilized myofibers, 2-way ANOVA followed by Bonferroni's multiple comparisons test was used.

Study approval. All experiments involving mice were approved by the IACUC at Cincinnati Children's Hospital Medical Center, approval number IACUC 2016-0069.

\section{Author contributions}

JDM and JQK wrote the manuscript. JQK, JH, MJB, JGB, JAS, JTM, YCJ, KS, and NG performed experiments. JDM, JD, DMB, and ZK provided experimental oversight, analyzed the data, and helped design the study.

\section{Acknowledgments}

JDM was funded by grants from the National Heart Lung and Blood Institute of the NIH, the Howard Hughes Medical Institute, and the Fondation Leducq. This work was also supported by funding from the American Heart Association (to JQK).

Address correspondence to: Jeffery D. Molkentin, Cincinnati Children's Hospital Medical Center, Howard Hughes Medical Institute, Molecular Cardiovascular Biology, 240 Albert Sabin Way, MLC 7020, Cincinnati, Ohio 45229 USA. Email: jeff.molkentin@cchmc.org.

1. Boncompagni S, et al. Mitochondria are linked to calcium stores in striated muscle by developmentally regulated tethering structures. Mol Biol Cell. 2009;20(3):1058-1067.

2. Denton RM. Regulation of mitochondrial dehydrogenases by calcium ions. Biochim Biophys Acta. 2009;1787(11):1309-1316.

3. Jouaville LS, Pinton P, Bastianutto C, Rutter GA, Rizzuto R. Regulation of mitochondrial ATP synthesis by calcium: evidence for a long-term metabolic priming. Proc Natl Acad Sci U S A. 1999;96(24):13807-13812.

4. Baughman JM, et al. Integrative genomics identifies MCU as an essential component of the mitochondrial calcium uniporter. Nature. 2011;476(7360):341-345

5. De Stefani D, Raffaello A, Teardo E, Szabò I, Rizzuto R. A forty-kilodalton protein of the inner membrane is the mitochondria calcium uniporter. Nature. 2011;476(7360):336-340.

6. Perocchi F, et al. MICU1 encodes a mitochondrial EF hand protein required for $\mathrm{Ca}(2+)$ uptake. Nature. 2010;467(7313):291-296.

7. Mallilankaraman $\mathrm{K}$, et al. MICU1 is an essential gatekeeper for MCU-mediated mitochondrial Ca(2+) uptake that regulates cell survival. Cell. 2012;151(3):630-644.

8. Plovanich M, et al. MICU2, a paralog of MICU1, resides within the mitochondrial uniporter complex to regulate calcium handling. PLoS One. 2013;8(2):e55785.

9. Sancak Y, et al. EMRE is an essential component of the mitochondrial calcium uniporter complex. Science. 2013;342(6164):1379-1382.

10. Hoffman NE, et al. SLC25A23 augments mitochondrial $\mathrm{Ca}^{2+}$ uptake, interacts with $\mathrm{MCU}$, and induces oxidative stress-mediated cell death. Mol Biol Cell. 2014;25(6):936-947.

11. Raffaello A, et al. The mitochondrial calcium uniporter is a multimer that can include a dominant-negative pore-forming subunit. EMBO J. 2013;32(17):2362-2376.

12. Pradhan RK, Qi F, Beard DA, Dash RK. Characterization of membrane potential dependency of mitochondrial Ca ${ }^{2+}$ uptake by an improved biophysical model of mitochondrial $\mathrm{Ca}^{2+}$ uniporter. PLoS One. 2010;5(10):e13278.

13. Pan $\mathrm{X}$, et al. The physiological role of mitochondrial calcium revealed by mice lacking the mitochondrial calcium uniporter. Nat Cell Biol. 2013;15(12):1464-1472.

14. Kwong JQ, et al. The mitochondrial calcium uniporter selectively matches metabolic output to acute contractile stress in the heart. Cell Rep. 2015;12(1):15-22.

15. Luongo TS, et al. The mitochondrial calcium uniporter matches energetic supply with cardiac workload during stress and modulates permeability transition. Cell Rep. 2015;12(1):23-34.

16. Mammucari C, et al. The mitochondrial calcium uniporter controls skeletal muscle trophism in vivo. Cell Rep. 2015;10(8):1269-1279.

17. Kanisicak O, Mendez JJ, Yamamoto S, Yamamoto M, Goldhamer DJ. Progenitors of skeletal muscle satellite cells express the muscle determination gene, MyoD. Dev Biol. 2009;332(1):131-141. 
18. Brennan KJ, Hardeman EC. Quantitative analysis of the human alpha-skeletal actin gene in transgenic mice. J Biol Chem. 1993;268(1):719-725.

19. Spriet LL. New insights into the interaction of carbohydrate and fat metabolism during exercise. Sports Med. 2014;44(supp1 1):S87-S96.

20. Kelley DE, Mandarino LJ. Fuel selection in human skeletal muscle in insulin resistance: a reexamination. Diabetes. 2000;49(5):677-683.

21. Glancy B, Willis WT, Chess DJ, Balaban RS. Effect of calcium on the oxidative phosphorylation cascade in skeletal muscle mitochondria. Biochemistry. 2013;52(16):2793-2809.

22. Bround MJ, et al. Cardiac Ryanodine Receptor (Ryr2)-mediated calcium signals specifically promote glucose oxidation via pyruvate dehydrogenase. J Biol Chem. 2016;291(45):23490-23505.

23. Vanhoutte D, et al. Thrombospondin expression in myofibers stabilizes muscle membranes. Elife. 2016;5.

24. Weisleder N, Zhou J, Ma J. Detection of calcium sparks in intact and permeabilized skeletal muscle fibers. Methods Mol Biol. 2012;798:395-410.

25. Kuznetsov AV, Troppmair J, Sucher R, Hermann M, Saks V, Margreiter R. Mitochondrial subpopulations and heterogeneity revealed by confocal imaging: possible physiological role? Biochim Biophys Acta. 2006;1757(5-6):686-691.

26. Lu X, Ginsburg KS, Kettlewell S, Bossuyt J, Smith GL, Bers DM. Measuring local gradients of intramitochondrial [Ca(2+)] in cardiac myocytes during sarcoplasmic reticulum $\mathrm{Ca}(2+)$ release. Circ Res. 2013;112(3):424-431.

27. Pasut A, Jones AE, Rudnicki MA. Isolation culture of individual myofibers their satellite cells from adult skeletal muscle. J Vis Exp. 2013;(73):e50074.

28. Schuh RA, Jackson KC, Khairallah RJ, Ward CW, Spangenburg EE. Measuring mitochondrial respiration in intact single muscle fibers. Am J Physiol Regul Integr Comp Physiol. 2012;302(6):R712-R719.

29. Powers C, Huang Y, Strauss A, Khuchua Z. Diminished exercise capacity and mitochondrial bc1 complex deficiency in tafazzin-knockdown mice. Front Physiol. 2013;4:74. 